\title{
Juan Luis RODRíGUEZ, Language and Revolutionary Magic in the Orinoco Delta
}

Olivier Allard

\section{OpenEdition}

Journals

Édition électronique

URL : https://journals.openedition.org/jsa/19713

DOI : 10.4000/jsa. 19713

ISSN : 1957-7842

Éditeur

Société des américanistes

Édition imprimée

Date de publication : 30 septembre 2021

Pagination : 241-245

ISSN : 0037-9174

\section{Référence électronique}

Olivier Allard, « Juan Luis Rodríguez, Language and Revolutionary Magic in the Orinoco Delta », Journal de la Société des américanistes [En ligne], 107-1 | 2021, mis en ligne le 30 septembre 2021, consulté le 03 septembre 2022. URL : http://journals.openedition.org/jsa/19713; DOI : https://doi.org/10.4000/jsa. 19713

Ce document a été généré automatiquement le 3 septembre 2022

Tous droits réservés 


\title{
Juan Luis RODRÍGUEZ, Language and Revolutionary Magic in the Orinoco Delta
}

\author{
Olivier Allard
}

\section{RÉFÉRENCE}

Juan Luis RODRíGUEZ, Language and Revolutionary Magic in the Orinoco Delta, Bloomsbury Academic (Bloomsbury Studies in Linguistic Anthropology), Londres/New York, 2021, 206 p., bibliogr., index, photos (en noir et blanc), carte, tabl.

1 Comment les Amérindiens ont-ils été affectés par l'État vénézuélien -État pétrolier par excellence - et par la «révolution bolivarienne » par laquelle Hugo Chávez a voulu transformer radicalement le pays au début du xxie siècle ? Juan Luis Rodríguez s'attaque à ces questions à partir d'une recherche menée dans le delta de l'Orénoque, où les Warao constituent l'essentiel de la population autochtone, et en privilégiant l'étude des discours. Sa thèse principale est que l'on a assisté à un double processus de traduction voire de "transduction" (terme qui permet de souligner que c'est une véritable transformation qui s'opère): le pétrole est converti en ressources étatiques, et ces ressources sont distribuées à la population, c'est-à-dire transformées en relations politiques. L'État vénézuélien est en effet un État-pourvoyeur, et l'accès à la richesse nationale s'apparente à un droit citoyen, comme d'autres spécialistes du pays l'ont également souligné. Le langage est au cœur de cette double opération de traduction qui, si elle se déroule en partie à un niveau central ou national (comme l'avait montré Coronil [1997]), repose aussi sur sa mise en œuvre dans les interactions entre politiciens locaux et populations locales - y compris autochtones. Juan Luis Rodríguez rappelle donc l'intérêt d'étudier les marges géographiques et politiques du Venezuela pour comprendre l'existence et la reproduction de l'État. Cette perspective lui offre ainsi l'occasion de reformuler le problème du clientélisme, en essayant de dépasser la représentation 
simpliste des Amérindiens comme des victimes qui voteraient aveuglément pour les hommes et les femmes politiques qui leur donnent de l'argent ou des biens : c'est en fait une relation plus complexe qui se noue entre les uns et les autres.

L'ouvrage est divisé en six chapitres, et on peut regretter que seuls deux d'entre eux soient proprement ethnographiques, ou du moins fondés sur une enquête de terrain au sens traditionnel. Dans les trois premiers, Rodríguez s'appuie en effet principalement sur des données textuelles, pour montrer que les Warao n'ont que tardivement été constitués comme un "public» (au sens d'Habermas). Il présente d'abord l'histoire du Delta Amacuro (entité administrative qui englobe plus ou moins le delta de l'Orénoque), puis le travail des missionnaires capucins au $\mathrm{xx}^{\mathrm{e}}$ siècle et leurs efforts pour étudier la langue warao, la traduire en espagnol et transformer les Warao en locuteurs hispanophones (condition de leur incorporation à la nation). Dans le troisième chapitre, Rodríguez souligne que, de simples spectateurs, les Amérindiens sont devenus au $\mathrm{xxI}^{\mathrm{e}}$ siècle les sujets d'une nouvelle forme de participation politique, dont la traduction de l'hymne national en warao est le signe - processus qui aurait pu être l'occasion d'une comparaison avec ce qui se passe dans les autres pays d'Amérique du Sud. Rodríguez souligne toutefois à juste titre que la plupart des Warao sont relativement indifférents aux usages de l'hymne vénézuélien traduit dans leur langue. C'est une question qu'il a donc principalement abordée en étudiant différentes traductions de l'hymne et des vidéos accessibles sur internet, où ce sont des écoliers non indigènes qui le chantent !

Les chapitres quatre et cinq constituent véritablement le cœur de l'ouvrage: en s'appuyant sur ses observations à Tucupita (la capitale régionale) et dans le bas delta, Juan Luis Rodríguez montre que l'anthropologie linguistique permet d'éclairer sous un jour nouveau le clientélisme politique. Les relations de clientèle acquièrent en effet leur signification par le biais d'un certain nombre d'actes de langage, notamment les promesses, qui les inscrivent dans la durée. Les réunions organisées par des responsables politiques s'ouvrent toujours par des salutations, qui sont également très importantes : elles permettent, comme dans le cas d'une ministre yek'wana qui s'adressait dans sa langue à un public warao, d'indexer une identité autochtone commune en dépit du défaut de compréhension réciproque (p. 102). Cependant, des discours prononcés par des hommes ou des femmes politiques warao dans leur langue, s'ils permettent certes de créer une atmosphère d'intimité, sont considérés comme une perte de temps par le public warao si aucune promesse n'y est faite. Ce sont donc aussi les Amérindiens qui demandent à l'État d'être magique et de continuer à distribuer des richesses en permanence (p. 114). Inversement, un don qui n'a pas été promis a des effets incertains, échouant souvent à créer un sentiment d'obligation chez les bénéficiaires. Rodríguez décrit le cas fascinant d'un générateur électrique donné en 2008 par un maire de l'époque, Amado Heredia, à une communauté du bas delta, alors que des élections allaient bientôt être organisées : par accident, il n'a pas été livré aux personnes auxquelles il avait été promis, mais à un leader de la faction qui soutenait la candidate opposée, Lizeta Hernández, finalement élue gouverneure de l'État... Les réunions et les discours se sont enchainés pour rétablir la signification du don, c'est-à-dire pour en faire un bienfait du maire reçu grâce à la fidélité de son principal soutien local, mais apparemment sans succès. Formuler le don comme tel et en faire le récit (framing and narrating the gift) en sont des dimensions absolument essentielles (p. 145), et ces analyses permettent de saisir tout l'intérêt d'une approche sémiotique des relations politiques. 
Dans le dernier chapitre, Rodríguez retourne à des données textuelles en analysant les interventions dans la presse régionale de différents candidats locaux, ainsi que le portrait que celle-ci dresse d'eux. Il est intéressant de voir, dans la continuité avec les chapitres précédents, que ces personnes s'attachent à définir leurs actions comme morales et mettent à distance un clientélisme toujours condamné : elles affirment distribuer des ressources publiques parce qu'elles sont généreuses et attribuent les calculs stratégiques à leurs adversaires; les femmes politiques soulignent plus particulièrement le lien affectif durable qu'elles ont développé avec leur électorat et avec la population locale en général (p. 154). Ce phénomène rappelle les descriptions que d'autres anthropologues ont $\mathrm{pu}$ faire des liens politiques en Amérique latine. Amado Heredia, qui est le seul Amérindien des quatre figures étudiées, a également cherché à établir une connexion entre son identité autochtone et le socialisme promu par Chávez à partir de 2005, faisant écho - quoi que sans s'y référer explicitement - à une longue tradition latino-américaine (p. 168).

5 En conclusion, Rodríguez défend l'idée que la révolution bolivarienne a en fait été une révolution rhétorique, avec une prolifération de discours prétendant qu'une transformation radicale du pays était en cours alors que le fonctionnement de l'État et sa base économique restaient inchangés (p.175). Il s'est effectivement produit une exacerbation du modèle de l'État-rentier dans les années 2000 et jusqu'au milieu des années 2010, grâce au cours très élevé du pétrole, ce qui ne contredit pas la place centrale que l'on peut accorder à la « rhétorique » dès lors que l'on considère l'État comme une entité qui existe dans et par le discours, en suivant une perspective bien établie en anthropologie.

Il est souvent délicat de discuter le travail d'un collègue dont on a plus ou moins partagé le terrain et les intérêts. Sur certains points, ma présentation du Delta Amacuro et des Warao serait probablement éloignée de celle qu'offre Rodríguez, et articulerait différemment ruptures et continuités. Tout d'abord, Rodríguez écourte un peu l'histoire régionale (voir p. 24-25, 179): les termes employés par les Warao pour désigner les positions d'autorité (kobenahoro, adapté de gobernador, kabitana, adapté de capitán, etc.) ne sont pas un héritage des missionnaires du $\mathrm{xx}^{\mathrm{e}}$ siècle mais plutôt de ceux du XvIII ${ }^{\mathrm{e}}$ siècle, car ils étaient déjà employés lors de l'arrivée des premiers (Turrado Moreno 1945, p. 56 ; Wilbert 1996, p. 75-76). De plus, des non-indigènes ont été présents dans le delta tout au long du XIX ${ }^{e}$ siècle, même s'ils sont probablement restés sur son pourtour (Level 1850). Ensuite, les missionnaires capucins espagnols qui sont arrivés dans les années 1920 ont joué un rôle éminemment politique et administratif, l'État leur ayant délégué une responsabilité étendue sur la population indigène de la région, et pas uniquement de prendre en charge leur éducation et leur évangélisation. De ce point de vue, la séparation entre l'histoire proprement politique du Delta Amacuro (chapitre 1) et l'histoire du travail linguistique des missionnaires (chapitre 2) est peut-être un peu forcée: la préoccupation de Rodríguez pour la sphère publique me semble largement une projection rétrospective, alors que la mise en valeur du territoire et la protection de la frontière sont sans doute plus importantes pour comprendre le rapport de nombreux acteurs de l'époque à la population locale.

7 D'autres différences concernent une histoire beaucoup plus récente. Rodríguez a travaillé avec des Warao qui votaient, et dont certains recevaient des avantages politiques, dès avant Chávez (les années 1960 pour les élections nationales, et les années 1990 pour les élections régionales): c'est une différence cruciale par rapport à mes interlocuteurs 
d'autres villages, dont beaucoup ne votaient pas auparavant, tout simplement parce qu'ils n'étaient pas inscrits sur les listes électorales et n'avaient même pas de carte d'identité. Ils n'en sont pas moins représentatifs de la population warao de la région. Par conséquent, Rodríguez minore peut-être certains des changements survenus sous Chávez : les droits politiques, et surtout l'accès à la richesse nationale qui en découle, ont été étendus à beaucoup de personnes qui en étaient de facto exclues jusqu'alors ${ }^{1}$. Pour de nombreux Warao, la révolution n'a pas été que rhétorique. Enfin, j’ai été aussi frappé que la presse régionale soit régulièrement lue dans la communauté du bas delta où Rodríguez a travaillé, alors que j'ai vécu dans des villages où seule une petite minorité de la population était alphabétisée (ce qui, en l'absence d'école, avait peu de chances de changer), et où les discours tenus essentiellement en espagnol dans la capitale avaient donc très peu d'effets. Il s'agit là d'une question importante pour comprendre le delta de l'Orénoque aujourd'hui : si les Warao ont longtemps été présentés comme formant quatre sous-groupes caractérisés par des cultures et des environnements distincts (Wilbert 1993), l'accès inégal aux ressources étatiques et à l'éducation constitue aujourd'hui un facteur peut-être plus déterminant de différenciation entre les villages et entre les personnes. C'est aussi pour cette raison que, malgré ces quelques réserves, il est très précieux que l'ouvrage de Juan Luis Rodríguez nous permette d'enrichir notre connaissance des Warao et des relations politiques dans le delta de l'Orénoque.

\section{BIBLIOGRAPHIE}

CORONIL Fernando

1997 The Magical State. Nature, Money, and Modernity in Venezuela, University of Chicago Press, Chicago.

LEVEL Andrés Eusebio

1850 Informe sobre el estado actual de los distritos de reducción de indígenas, Alto Orinoco, Central y Bajo Orinoco y medidas que reclaman, Imprenta de Diego Campbell, Caracas.

TURRADO MORENO Angel

1945 Etnografia de los indios guaraunos, Lit. y Tip. Vargas (Tercera Conferencia Interamericana de Agricultura, Serie nacional, 15), Caracas.

WILBERT Johannes

1993 Mystic Endowment. Religious Ethnography of the Warao Indians, Harvard University Center for the Study of World Religions (Religions of the World), Cambridge (MA).

WILBERT Johannes

1996 Mindful of Famine. Religious Climatology of the Warao Indians, Harvard University Center for the Study of World Religions (Religions of the World), Cambridge (MA). 


\section{NOTES}

1. Dans le "Municipio Antonio Díaz », dont la population est presque entièrement indigène (et où Juan Luis Rodríguez et moi-même avons tous les deux travaillé), il y a eu trois fois plus de votes exprimés aux élections municipales de 2008 et de 2013 qu'à celles de 2000 (suivant les données disponibles sur le site du Conseil national électoral, http:// www.cne.gob.ve/, consulté le 25 mai 2021). C'est là qu'Amado Heredia a été élu maire à plusieurs reprises.

\section{AUTEURS}

\section{OLIVIER ALLARD}

LAS, EHESS 